\title{
Seroprevalence of hepatitis E in a tertiary care hospital
}

\begin{abstract}
Background and Objectives: In this study, we aimed to evaluate the seroepidemiology of HEV in a tertiary care hospital including date of eight years.

Methods: Medical records of the patients who have been tested for HEV serology between January 2006 and July 2013 were reviewed. A serum sample of the patients was tested for HEV IgM and IgG antibodies by ELISA method (DIA-Pro, Italy).

Results: A total of 6391 patients' HEV serology results was examined. The average age of the patients was 49.1 years ( $0-85$ year) and $3337(52.2 \%)$ of them were females. Of the 6391 patients $4735(74.1 \%)$ were over 18 years-old. Out of 6391 patients $205(3.2 \%)$ were found positive for HEV (IgM, $\operatorname{IgG})$. Of 205 positive results 137 (66.8\%) were positive for $\operatorname{IgG}$ and $68(33.2 \%)$ were positive IgM and $182(86.8 \%)$ of them were over 18 years old. The high seropositivity was detected in patients from gastroenterology clinic $(29.8 \%)$. Seropositivity of IgG among adults was found significantly higher than children $(\mathrm{p}<0.001)$. No statistically significant difference was detected for IgM seropositivity among adults and children.
\end{abstract}

Conclusion: The HEV seropositivity rate $(2.1 \% \operatorname{IgG}$ and $1 \% \operatorname{IgM})$ detected in our study was similar to the results reported from northern and western part of the country.

Keywords: seroprevelance, hepatitis E, Turkey
Volume 2 Issue 2 - 2015

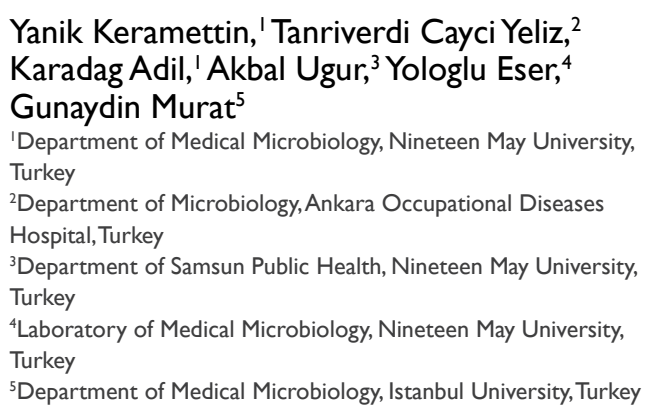

Correspondence: Yeliz Tanriverdi Cayci, Department of Microbiology, Ankara Occupational Diseases Hospital,Ankara, Turkey,Tel +9050569|2125, Email yeliztanriverdi@gmail.com

Received: March 10, 2015 | Published: April 29, 2015

\section{Introduction}

A large outbreak of acute viral hepatitis in New Delhi in 1955-56 was retrospectively found to be due to an agent called enteric non-A, non-B hepatitis. This agent was later named hepatitis E virus (HEV). ${ }^{1}$ $\mathrm{HEV}$ is a non-enveloped, positive-sense, single-stranded RNA virus with icosahedral symmetry. $\mathrm{HEV}$ is a leading cause of acute and fulminant hepatitis in developing countries. ${ }^{2,3} \mathrm{HEV}$ has been considered an important disease associated with travel to a diseaseendemic area. However, an increasing number of sporadic Hepatitis E cases not associated with travel have been reported from developing countries, today. ${ }^{4}$ The HEV target population is young to middle aged adults, 15 to 40 years of age. The clinical symptoms represent as acute viral hepatitis and include jaundice, malaise, anorexia, abdominal pain, fever and hepatomegaly. ${ }^{5}$ Compared with HAV that is transmitted via fecal-oral route like HEV; HAV has a $10-20 \%$ secondary attack rate among household contacts, HEV has relatively low infectivity, with a secondary attack rate of about $2 \%{ }^{6}$ Increased morbidity and mortality is observed in chronic liver disease patients super infected with HEV. The main clinical feature is its increased incidence and severity in pregnant women, with mortality rates of $15-20 \%$. And an endotoxin mediated hepatocyte injury was proposed for the severity of the HEV in pregnancy. ${ }^{1}$

There are four genotypes of HEV. Genotype 1 is identified from disease-hyperendemic such as Africa and southern Asia; genotype 2 from Mexico and West Africa; genotype 3 from developed countries such as North America, Europe, and Japan; and genotype 4 from eastern Asia and India. ${ }^{4}$ The virus is widely distributed in nature and it is detected in animals, both domestic and wild. ${ }^{7}$ Genotypes 1 and 2 are mostly isolated from humans, however genotypes 3 and 4 have also been isolated from swine and other animals. ${ }^{8}$ In developed countries, HEV Seroprevalence rates were $0.4 \%-3 \%$. Whereas fecal-oral HEV transmission is predominantly in developing countries, other routes such as consumption of different meat have been demonstrated. Parenteral transmission was first suggested, but not demonstrated. ${ }^{2}$ It is difficult to differential diagnosis of HEV from infectious and noninfectious liver diseases. The knowledge of hepatitis E seroepidemiology in regions should be helpful for clinicians in differential diagnosis. In this study we aimed to describe the seroepidemiology of HEV infection in a tertiary care hospital including data of eight years.

\section{Materials and methods}

This was a hospital-based study conducted between January 2006 and July 2013 in the Ondokuz Mayis University, Medical Faculty Teaching Hospital, which is a tertiary care center that provides medical services to the middle Black sea region. Medical records of patients whom serum samples had been studied for HEV were reviewed, retrospectively. HEV IgM and IgG antibodies have been tested by ELISA method (DIA-pro, Italia) according to the recommendation of the manufacturer. Fisher's exact test was used for the comparison of $\operatorname{IgG}$ and IgM seropositivity among adults and children. Chi square test was used for the statistical evaluation of the clinics for seropositivity rates.

\section{Results}

A total of 6391 patients' HEV serology results was examined. The average age of the patients was 49.1 years $(0-85$ year) and $3054(47.8 \%)$ of them were males and $3337(52.2 \%)$ of them were females. Of the 6391 patients $1656(25 \%)$ were under 18 years-old 
and $4735(74.1 \%)$ over 18 years old. Out of 6391 patients $205(3.2 \%)$ were found positive for HEV ( $\operatorname{IgM}, \operatorname{IgG})$. Of 205 positive results $137(66.8 \%)$ were positive for IgG and $68(33.2 \%)$ were positive IgM. $182 \mathrm{HEV}$ seropositivity was found in the population over 18 years old that tested for HEV. In the population of less than 18 years-old, 23 of them had seropositivity for HEV (Table 1).

Table I Anti HEV IgG and IgM seropositivity

\begin{tabular}{llll}
\hline Marker & Age Group & & \\
& $>18 \mathrm{n:} 1656(\%)$ & $<18 \mathrm{n:} 4735(\%)$ & Total n: 639। (\%) \\
Anti HEV IgG & $122(59.5 \%)$ & $15(7.3 \%)$ & $137(66.8 \%)$ \\
Anti HEV IgM & $60(29.3 \%)$ & $8(3.9 \%)$ & $68(33.2 \%)$ \\
Totally & $182(88.8 \%)$ & $23(11.2 \%)$ & $205(100 \%)$ \\
\hline
\end{tabular}

$\mathrm{n}$ : Number of patients

The most of the patients that tested for HEV were from pediatrics wards $(31 \%)$. The high seropositivity were detected in patients from gastroenterology clinic $(29.8 \%)$ followed by internal medicine clinic $(16.6 \%)$ and pediatric clinic (13.3\%) (Table2).

Table 2 Anti HEV seropositivity rates for the clinics

\begin{tabular}{|c|c|}
\hline Clinic & $\begin{array}{l}\text { Anti HEV } \\
\text { Seropositivity n: } \\
\text { 205(\%) }\end{array}$ \\
\hline Gastroenterology N: I 294 & $62(29.8 \%)$ \\
\hline Internal Medicine N: 773 & $34(16.6 \%)$ \\
\hline Pediatric N: 1656 & $27(\mid 3.3 \%)$ \\
\hline Emergency N: 322 & $\mathrm{II}(5.4 \%)$ \\
\hline Surgery N: 32I & $\mathrm{II}(5.4 \%)$ \\
\hline Chest Diseases N: 250 & $\mathrm{II}(5.4 \%)$ \\
\hline Infectious Diseases N: 373 & $10(4.9 \%)$ \\
\hline Obstetrics and Gynecology N: 544 & $10(4.9 \%)$ \\
\hline Hematology N: 269 & $8(3.9 \%)$ \\
\hline $\begin{array}{l}\text { Physical Therapy and Rehabilitation } \\
\text { N: } 127\end{array}$ & $6(2.9 \%)$ \\
\hline Other* N: 462 & $15(7.5 \%)$ \\
\hline
\end{tabular}

$\mathrm{N}$, number of samples are collected from each type of clinic; $\mathrm{n}$, positive samples; *: Neurosurgery, Dermatology, Thoracic Surgery, Cardiology, Otolaryngology, Neurology, Orthopedic surgery, Psychiatric

The anti- HEV IgG seropositivity rates among the age groups was presented in Table 3. Of the 68 seropositive patients for IgM, 4 of them were pregnant. The medical records of these pregnant patients were examined. And it was determined that none of them had fulminant HEV.

Table 3 Distribution of Anti-HEV IgG and IgM seropositivity among ages

\begin{tabular}{lll}
\hline Age Group (N) & Anti HEV IgG n(\%) & Anti HEV IgM n(\%) \\
\hline $0-9(1263)$ & $11(8.0 \%)$ & $8(11.8 \%)$ \\
$10-18(393)$ & $5(3.6 \%)$ & $5(7.4 \%)$ \\
$19-29(1512)$ & $8(5.8 \%)$ & $9(13.2 \%)$ \\
$30-39(923)$ & $14(10.2 \%)$ & $8(11.8 \%)$ \\
$40-49(727)$ & $26(19.0 \%)$ & $6(8.8 \%)$ \\
$50-59(675)$ & $18(13.1 \%)$ & $9(13.2 \%)$ \\
$60-69(470)$ & $26(19.0 \%)$ & $4(20.6 \%)$ \\
$70-79(343)$ & $26(19.0 \%)$ & $9(13.2 \%)$ \\
$>80(85)$ & $3(2.2 \%)$ & 0
\end{tabular}

$\mathrm{N}$, number of samples are collected from each type of clinic; $n$, positive samples

Seropositivity of $\mathrm{IgG}$ among adults was found significantly higher than children $(\mathrm{p}<0.001)$. No statistically significant difference was detected for IgM seropositivity among adults and children $(\mathrm{p}<0.000)$. Significant difference was obtained for the seropositivity among clinics $(\mathrm{p}<0.001)$.

\section{Discussion}

In developing countries, HEV is transmitted through the fecaloral route, mainly by the use of water contaminated with sewage disposal. The another transmission route for HEV is via by food, in Japan acute hepatitis E was diagnosed in patients who ate raw or undercooked pig liver and intestine, liver and deer meat contaminated by the virus. Conversely to hepatitis A virus (HAV) infection, secondary transmission of HEV infection person to person is an uncommon event. Transmission of HEV via blood transfusion has been documented in several countries, including Saudi Arabia, Japan, the UK and France. ${ }^{2,9}$

In the diagnosis of HEV, the main method is enzyme immune assays. And most of them for HEV infection are based on either recombinant HEV proteins or synthetic peptides. It was mentioned that these assays have varied significantly. Myint et al., ${ }^{10}$ evaluated assays for diagnosis of HEV and observed that the sensitivity of all serological assays was greater for symptomatic than for asymptomatic HEV infections.

The HEV seropositivity in our country varies by regions. AntiHEV IgG seropositivity was detected in $20.6 \%$ of hemodialysis patients in a southern province of Turkey. ${ }^{11}$ Eker et al., ${ }^{12}$ reported HEV seropositivity rate as $2.4 \%$ in the northwest of Turkey. Thomas et al., ${ }^{13}$ determined anti-HEV seropositivity as $5.9 \%$ in a study included data from five different provinces in Turkey, furthermore they mentioned that seropositivity was higher among adults over 30 years old. In 1046 people aged from 15 to 75 who admitted hospital in Ankara, anti-HEV seropositivity was determined in 40 (3.8\%) of them, and seropositivity was the highest between the ages of 30 and 60 years. ${ }^{14}$ In our study, we determined that HEV seropositivity was higher in adults over 40 years old. In a study conducted in Malatya, seropositivity of 600 people was reported to be as $9.8 \% .{ }^{15}$ Olcay et al., ${ }^{16}$ investigated anti-HEV seropositivity in three distinct regions (Manisa, Elmadag and Diyarbakir) of Turkey, and they reported 
that the overall anti-HEV antibody Seroprevalence rate was $6.3 \%$ (57/910). It was $2.7 \%$ in Elmadag/Ankara, $3.8 \%$ in Manisa and $11.7 \%$ in Diyarbakir. They mentioned that there was a significant difference between the rates of Diyarbakir and the other two regions. In epidemic countries like India, peak Seroprevalence levels were revealed as $33-40 \%$ of the adult population. ${ }^{9} \mathrm{Li}$ et al., ${ }^{8}$ reported that average anti-HEV IgG Seroprevalence of the Chinese population was $43 \%$, and HEV infection was endemic in southern China and might have been so for at least 60 years. The prevalent genotype was 4 . In California (USA), among blood donors, anti-HEV Seroprevalence was reported between 1.2-1.4 \%, and in a study conducted in the USA, interestingly, the highest prevalence was found among intravenous drug users $(23 \%)$ and homosexual men (16\%). The seropositivity of HEV in blood donors and healthy population in European countries was determined between 1-2.6\%. ${ }^{1}$ Although seropositivity rates were not high in childhood population, anti-HEV tests were much ordered from pediatricians. In our opinion, the reason for this should be the difficulty of diagnosis of HEV infection from other infectious diseases.

In our study, anti-HEV IgG and IgM seropositivities were found as $2.1 \%$ and $1 \%$, respectively. The rate of anti-HEV seropositivity was lower in our study. We observed that HEV seropositivity was higher in patients from gastroenterology, internal medicine and pediatric clinics than the other clinics. This distribution is expected result, because of the clinical symptoms of HEV and patients admitted to these clinics.

\section{Acknowledgments}

None.

\section{Conflicts of interest}

Authors declare that there is no conflict of interest.

\section{References}

1. Chandra V, Taneja S, Kalia M, et al. Molecular biology and pathogenesis of hepatitis E virus. J Biosci. 2008;33(4):451-464.

2. Colson P, Coze C, Gallian P, et al. Transfusion-associated hepatitis E, France. Emerg Infect Dis. 2007;13(4):648-649.

3. Worm HC, van der Poel WH, Brandstatter G. Hepatitis E: an overview Microbes Infect. 2002;4(6):657-666
4. Lewis HC, Molbak K, Reese C, et al. Pigs as Source of MethicillinResistant Staphylococcus aureus CC398 Infections in Humans, Denmark. Emerg Infect Dis. 2008;14(9):1383-1389.

5. Smith JL. A review of hepatitis E virus. $J$ Food Protect. 2001;64(4):572-586.

6. Labrique AB, Thomas DL, Stoszek SK, et al. Hepatitis E: an emerging infectious disease. Epidemiol Rev. 1999;21(2):162-179.

7. Meng XJ, Purcell RH, Halbur PG, et al. A novel virus in swine is closely related to the human hepatitis E virus. Proc Natl Acad Sci USA. 1997;94(18):9860-9865.

8. Li RC, Ge SX, Li YP, et al. Seroprevelance of Hepatitis E virus infection, rural southern People's of Republic China. Emerg Infect Dis. 2006;12(11):1682-1688.

9. Pelosi E, Clarke I. Hepatitis E: a complex and global disease. Emerg Health Threats J. 2008; 1:e8.

10. Myint KS, Endy TP, Gibbons RV, et al. Evaluation of diagnostic assays for hepatitis E virus in outbreak settings. $J$ Clin Microbiol. 2006;44(4):1581-1583.

11. Ucar E, Cetin M, Kuvandik C, et al. Hepatitis E virus seropositivity in hemodialysis patients in Hatay province, Turkey. Mikrobiyol Bul. 2009;43(2):299-302.

12. Eker A, Tansel O, Kundurucilar H, et al. The epidemiology of hepatitis E virus infection in adults in Edirne. Mikrobiyol Bul. 2009;43:251-258.

13. Thomas DL, Mahley RW, Badur S, et al. Epidemiology of hepatitis E virus infection in Turkey. Lancet. 1993;341(8860):1561-1562.

14. Cesur S, Akin K, Dogaroglu I, et al. Hepatitis a and Hepatitis E Seroprevalence in the Adults of Ankara Region. Mikrobiyol Bul. 2002;36(1):79-83.

15. Otlu B, Durmaz R. Seasonal Distribution of Hepatitis E Virus Seropozitifity. Journal of Inonu University Medical Faculty. 2001;8(4):181-183.

16. Olcay D, Eyigun CP, Ozguven SK, et al. Anti-HEV antibody prevalence in three distinct regions of Turkey and its relationship with age, gender, education and abortions. Turk J Med Sci. 2003;33:33-38. 